\title{
Objective and subjective nutritional assessment of patients with cancer in palliative
} care.

\begin{abstract}
This study aimed to evaluate the nutritional status of patients with cancer in palliative care and to examine the interrelationship between objective and subjective nutritional assessment measures. Patients' nutritional status in a palliative care unit of a Malaysian government hospital and a hospice facility were assessed using anthropometric measurements, weight loss at 1/6 months, and the scored patient-generated subjective global assessment (PG-SGA). Moderate-to-severe malnutrition was observed in a range from $31 \%$ to $69 \%$ using both measurements. Common nutritional impact symptoms were pain, xerostomia, and anorexia. Patient-generated subjective global assessment scores were significantly correlated with anthropometric measurements ( $\mathrm{P}<.050)$. The PG-SGA is equally informative as objective indicators and is recommended as a quickly applied tool for nutritional status assessment of patients with cancer in palliative care.
\end{abstract}

Keyword: Anthropometry; Cancer; Malnutrition; Nutritional assessment; Palliative care; Patient-generated subjective global assessment. 